University for Business and Technology in Kosovo

UBT Knowledge Center

UBT International Conference

2018 UBT International Conference

Oct 27th, 1:30 PM - 3:00 PM

\title{
NB-IoT: A Network slice for Massive loT
}

\author{
Xhafer Krasniqi \\ University for Business and Technology, xhafer.krasniqi@ubt-uni.net
}

Follow this and additional works at: https://knowledgecenter.ubt-uni.net/conference

Part of the Computer Sciences Commons, and the Digital Communications and Networking Commons

\section{Recommended Citation}

Krasniqi, Xhafer, "NB-IoT: A Network slice for Massive IoT" (2018). UBT International Conference. 99. https://knowledgecenter.ubt-uni.net/conference/2018/all-events/99

This Event is brought to you for free and open access by the Publication and Journals at UBT Knowledge Center. It has been accepted for inclusion in UBT International Conference by an authorized administrator of UBT Knowledge Center. For more information, please contact knowledge.center@ubt-uni.net. 


\title{
NB-IoT: A Network slice for Massive IoT
}

\author{
Xhafer Krasniqi ${ }^{1}$ \\ UBT - Higher Education Institution, Lagja Kalabria, 10000 p.n., Prishtinë, Kosovo \\ xhafer.krasniqi@ubt-uni.net
}

\begin{abstract}
Network virtualisation is a concept that has been around for some time now, but the concept of network slicing is more recent and only started to be mentioned in the context of 5G. In essence, network slicing is a specific form of network virtualisation that enables sharing of physical resources by multiple logical network slices used to serve different applications and use cases. One such a use case would be to allocate network resources to small things, e.g. sensors and very low power consumption objects. To allow these small objects access to network resources or to a slice of such resource, a new technology known as NB-IoT is being developed by different industry players.

This paper provides an overview of NB-IoT technology and Network Slicing concept and how this concept is applied to allocate network resources to very tiny objects and things. Finally, this paper will analyse the overall performance of NB-IoT in terms of power saving and battery life using eDRX saving mechanism.
\end{abstract}

Keywords: Network Slicing, IoT, NB-IoT, 5G

\section{Introduction}

Network slicing is a virtual network architecture, built on the principles of NFV (Network Functions Virtualization) and SDN (Software-Defined Networking). This technology enables slicing of a single, physical network into multiple virtual networks, where a slice represents an isolated and independent virtualized end-to- end network. The virtual networks are then customized based on the specific applications and services and as such making this technology play a very critical role in $5 \mathrm{G}$ [4].

Within a 5G system, the network slicing can offer connectivity for IoT devices, e.g. smart meters and water meters, through a slice with high availability, a specified latency, data rate and security and also through a different network slice with high throughput, fast data speeds and low latency. The main benefit of the network slicing is to allow operators to provide networks as a service, which results in lowering operational and capital expenses and at the same time improving operational efficiency and time to market. Though network slicing is mainly applied to the core network, it could also be applied in the RAN and transport network by logically abstracting physical radio resources, such as spectrum and physical hardware.

While Network Slicing technology can interwork with a number of wireless technologies to support the connectivity of IoT devices, such as WiFi, ZigBee, Bluetooth, a significant proportion of IoT devices will be connected using low power wide area networks, such as LoRa, SigFox, LTE-M and NB-IoT.

NB-IoT, as the key topic of this paper, is a new radio technology that is also known as a subset of network slicing. NB-IoT can be treated as a pre-configured network slice with many different tenants sharing the same NB-IoT network. This technology supports massive machine-type communications with the focus on coverage improvement and power saving as well as 
deployment simplification. By coverage improvement in this case is meant the capability to connect devices in deep and awkward locations where the signal penetration was not easy or was not possible with other technologies.

This paper focuses on the limitations of eDRX (extended Discontinuous Reception), the power saving mechanism for NB-IoT, and the proposals to overcome these limitations [3]. In addition, this paper also analyses how eDRX power saving compares with the use of different clocking for frequency synchronisation and data reporting by IoT devices

\section{Network Slicing Technology}

Network slicing is a form of virtual network architecture using the same principles behind software defined networking (SDN) and network functions virtualisation (NFV) in fixed networks that enable greater network flexibility by allowing traditional network architectures to be partitioned into virtual elements that can be linked through software [4].

Network slicing allows multiple virtual networks to be created on top of a common shared physical infrastructure as shown in the figure 1 . These slices that comprise an independent set of logical network

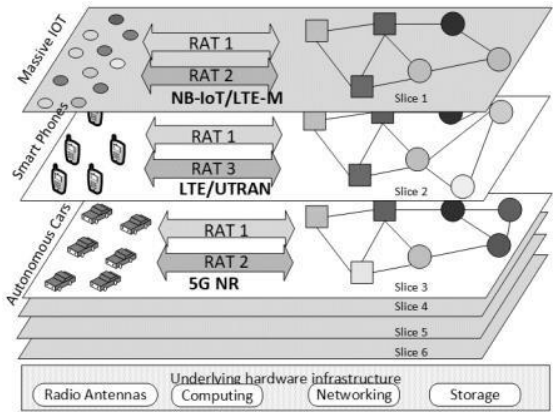

functions are customised to meet the specific needs of applications, services, devices, customers or operators. Functions such as speed, capacity, connectivity and coverage will be allocated to meet the particular demands of each use case, but functional components

may also be shared across different network slices.

Figure 1, Network slicing concept A specific network slice with an enhanced coverage capability can be allocated to massive IoT and the most suitable technology to enable this enhanced coverage is NB-IoT [6].

In order to enhance the performance of each slice, network slices are completely isolated so that no slice can interfere with the traffic in another slice. The isolation of slices reduces the risk of introducing and running new services and as such impacting the performance and also helps containing a potential cyber-attack on one slice and not affecting other slices.

Network slicing is part of the evolution towards $5 \mathrm{G}$ and will be added to $5 \mathrm{G}$ architecture given that many $5 \mathrm{G}$ services will run as network slices and in particular those network slices used for critical applications [5].

Network slices can be grouped into different types based on the abstraction of characteristics of the services, though the methodology to define and categorize the slices can vary between the network slice providers.

Abstraction of different characteristics can be roughly grouped into three categories: performance, functional and operational. The categorization of Network Slices helps network slicing users and providers have a common understanding of Network Slicing attributes and requirements when using or providing the network slicing service 


\section{Network Slicing Applications}

Network slicing can apply to a wide range of industry sectors and vertical markets such as health and wellness, industry internet, automation, financial etc., but the two main sectors where network slicing have found wide application are smart city and utilities sector.

The requirements for these two sectors go beyond what LTE can provide and this is where NBIoT, as the main topic of this paper, fits and enhances the coverage to provide not only the necessary support for the critical machine type communication (MTC) applications of energy grid protection and control, but also to support the massive volume of MTC type applications of the emerging smart metering [4].

\section{NB-IoT Technology}

NB-IoT is an IoT transmission technology that is being developed for low data rate services that make over $67 \%$ of total IoT services [2]. This high percentage of low data rates was a clear indication to the industry that something had to be done to develop a reliable cellular-based technology for IoT data. This is also to say that NB-IoT belongs to the category of long-distance transmission known as LPWAN (Low Power Wide Area network), as opposed to short-distance transmission technologies, such as ZigBee, WiFi, Bluetooth etc., that mainly apply in smart homes. Furthermore, NB-IoT belongs to categories of technologies that work in licensed spectrum as opposed to LoRa and Sigfox that are also low power wide area networks, but operate in unlicensed spectrum.

Main application of NB-IoT is smart metering and intelligent environment monitoring where millions of different sensors collect and generate data which can be analysed and act upon accordingly

\section{NB-IoT architecture}

NB-IoT is a very promising technology since is based on a reliable cellular communications network and can support connections of billions of IoT devices with ultra-low power consumption requirements and within a wide area coverage. NB-IoT architecture is based on LTE architecture, as shown in figure 2, though it is not backward compatible with existing 3GPP devices. The technology has some additional architecture elements and interfaces added to the EPC required to support IoT low data rate and low power consumption devices. This architecture is designed in such a way to support low data rate IoT IP and non-IP data [7]. While IoT Non-IP or small IP data can go over a control channel and IoT IP data can go over a normal LTE data channel, for the non-IP data, using the normal LTE data channels is considered to be inefficient since it requires complex procedures for bearer setup and underused for small infrequent data. Therefore, a new functionality, SCEF (Service Capability Exposure Function) and a couple of new interfaces, T6a and S6t are added to the NB-IoT architecture just for IoT non-IP data [7].

SCEF provides a means to securely expose $3 \mathrm{GPP}$ services and capabilities to IoT applications and serves as a bridge between MME and IoT applications to allow transfer of non-IP IoT data between IoT devices andservice/application servers Figure 2, NB-IoT architecture

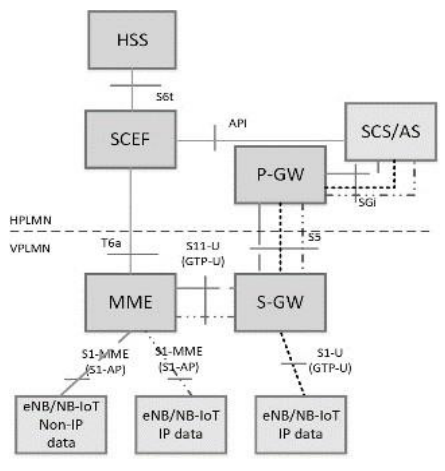




\section{NB-IoT Key Features}

Specific capabilities of NB-IoT to address the main IoT requirements are as following:

Lower Power Consumption: This capability is achieved through two different power consumption control mechanisms, PSM (Power Saving Mode) and eDRX that were added to 3GPP releases 12 and 13 .

PSM is a battery saving mechanism that gives more control to UEs in terms of power management required for its applications by allowing IoT devices to go idle without having to re-join the network when they wake up [10].

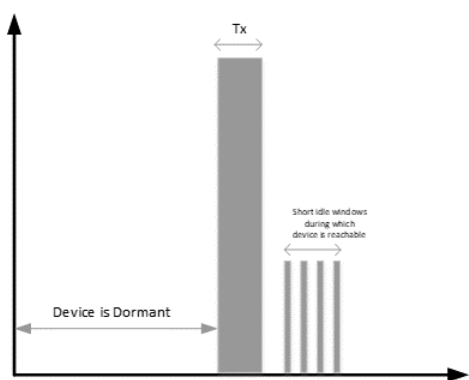

Figure 3, PSM operation

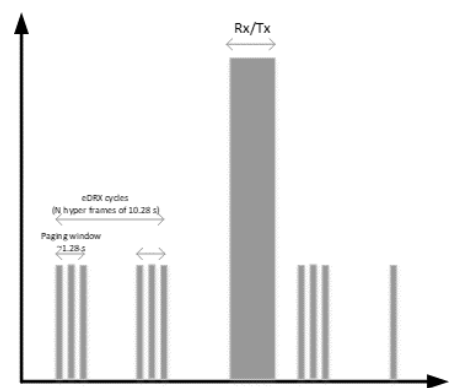

Figure 4, eDRX operation

PSM allows UEs to adjust wake-up periods and offset them as much as possible. It is similar to power-off but the devices remain registered on the network.

On the other side, eDRX that is another battery saving mechanism required for NB-IoT allows IoT devices to remain inactive for longer periods. More on this mechanism in the next section on eDRX with the focus on eDRX limitations and the proposed outcomes [10].

Enhanced Coverage: This is a feature that allows deep indoor coverage achieved through the addition of new information elements over S1-AP protocol and information elements for paging assistance to optimize the paging over S1-AP protocol. To achieve the enhanced coverage, NBIoT adopts two mechanisms, such as retransmission (200 times) and low frequency modulation. Retransmission mechanism improves the coverage by exploiting time diversity and the possibility to combine the replicated transmissions. NB-IoT can reach up to $164 \mathrm{~dB}$ coverage power which is higher than current LTE and helps to reach more difficult locations.

Transmission Mode: Even though NB-IoT is a new radio technology, the development of it is based on LTE. The actual NB-IoT physical layer radio bandwidth is $200 \mathrm{KHz}$, but like LTE on the downlink it adopts QPSK modulation and OFDMA technology while on the uplink adopts PSK or QPSK modulation and SC-FDMA technology [2]

Low Latency Sensitivity: Due to rare reporting and the need for retransmission of mass data, the latency increases and make this technology tolerable to higher latencies. Currently the tolerable latency in 3GPP IoT is $10 \mathrm{~s}$, but $6 \mathrm{~s}$ for maximal coupling losses can be also supported. This latency is much higher when compared with LTE latency that is in the range of $10 \mathrm{~ms}$ [8].

Other features supported by NB-IoT are Cell Reselection in idle mode, rate control and additional power class, $14 \mathrm{dBm}$ (class 6) in addition to classes $23 \mathrm{dBm}$ and $20 \mathrm{dBm}$ that are also supported by LTE 


\section{NB-IoT serving as a Network Slice}

As indicated earlier, NB-IoT is a subset of Network Slicing or a pre-configured network slice suitable for massive IoT devices. It is as an IoT connectivity technology that leverages the network slicing to enhance the accuracy of service provision by allocating different slices to specific use cases and to different IoT users. These different slices may have special charging and control functions that would make the network management easier and can lead to faster deployment.

An example could be to allocate a network slice for just monitoring the real-time status of public transport in a city and transmit this information via NB-IoT to a data analysis centre in order to enhance the traffic efficiency and lower the cost of city services. Other examples where NB-IoT would leverage network slicing in smart cities sector would be to enable smart parking, smart waste management etc.

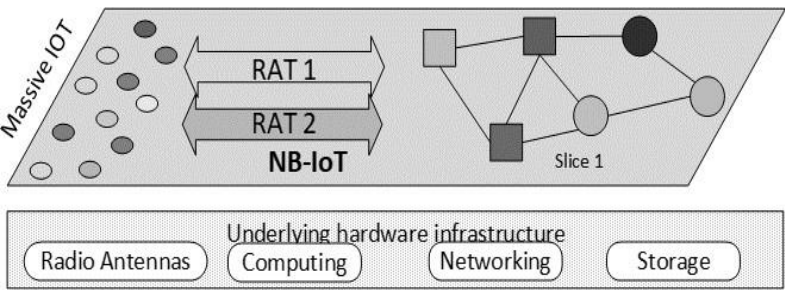

Figure 5, NB-IoT as a network slice As for other examples in other sectors, e.g. utility sector, where network slicing could play a critical role is smart meters and aggregator gateways to improve the power supply based on more accurate power consumption maps and as such control the power production

\section{eDRX Power Consumption Control}

eDRX is an enhanced version of DRX (Discontinuous Reception), a well-known power control mechanism in LTE that stops the receiver receiving any data during the sleeping mode. The eDRX has also the similar purpose, to put the receiver in the sleeping mode, but the sleeping period in eDRX gets longer, as shown in the figure 6 , due to more rigorous power consumption requirements for IoT. In essence, eDRX extends the sleeping duration of both types of DRX modes, the idle mode (Paging mode)

and connected mode (C-DRX) to further save energy as shown in figure 6 .

eDRX cycles are used to provide UEs with longer inactive periods between reading, paging or controlling channels and allows MME to page the device only at valid paging occasions as opposed to unconditional paging of UE that would happen normally. Paging occasions are defined based on eDRX parameters, Paging Time Window (PTW) and eDRX length duration. These two parameters

are sent to the eNB by the MME during paging.

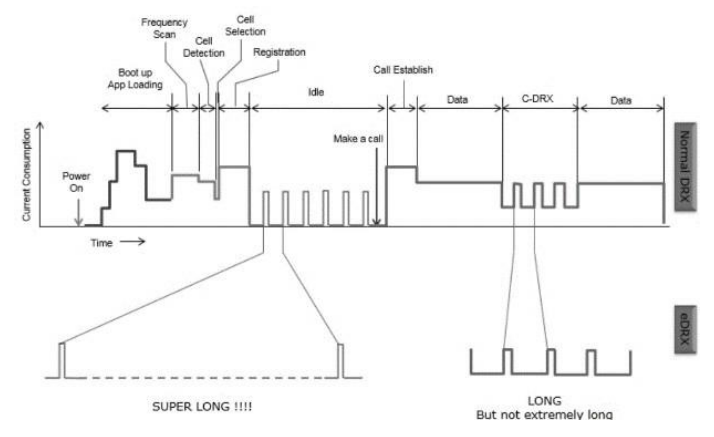

Figure 6, eDRX vs. DRX mechanism

During the idle mode the device just monitors the control channel for any paging message based on eDRX cycle, and then goes to sleep, during which period everything in a device sleeps, including the accurate crystal clock, except a low power clock. This low power clock stays on, but is not an accurate clock and can lead to inaccuracies during the frequency synchronisation period by waking up the device either too early and increase the power consumption or too late and miss the scheduled paging 


\section{NB-IoT Power Consumption Calculation}

Reducing the power consumption is the main requirement for NB-IoT to ensure long battery life of the IoT devices. In order to achieve more accurate calculation of power consumption, four different operating states should be considered individually: transmission, receiving, idle and sleeping states.

Transmitting state: During this state the IoT device can transmit reports, but certain events have to happen beforehand in sequence; downlink synchronisation obtained by the device happens during the synchronisation period, system information during the physical broadcast channel $(\mathrm{PBCH})$ period and the uplink synchronisation which is achieved during the random-access period. Following the report transmission, the device can continue with the monitoring of the control channel based on the eDRX parameters, or it can go to sleep.

Receiving state: During this state, the device has to be switched on for a period of time defined by eDRX as the time during which the physical download control channel (PDCCH) is granted to the device in order to check for the paging and after this can go to sleep.

Idle state: This state that is also known as Paging state, is the period during which the device has to monitor whether the network is sending any paging message to it. The device has to spend some battery to run in this state.

Sleeping state: This is the state that is also known as discontinuous reception during which the device sleeps and the receiver of the device does not receive any data. In this state, most of the device circuits are off to save battery, except a low power clock that not necessarily is very accurate

\section{eDRX Power Consumption Control analysis}

To avoid inaccuracies during the synchronisation period resulting from inaccurate low power clock, the receiver of the device wakes up and goes to sleep few times during the sleeping mode based on the eDRX cycle. These wake ups are very short, but frequently and as such the accurate crystal clock gets activated and it does not go to sleep when the receiver goes to sleep resulting in increased power consumption. Accurate crystal clock is active only during the active time and not during the sleeping time, but because the receiver wakes up frequently and stays on for a very short time during the eDRX off period, the accurate clock has no chance to turn off and therefore increases the power consumption. For the accurate crystal clock to go to sleep during the off period, the sleeping periods of the receiver during the off time have to be longer in order to have fewer wake ups and fewer active times as suggested in [3]. However, this is not easy to achieve since the active time has to be proportional to the sleeping time during the off period of eDRX. One possible solution as suggested in [3] is to change the duration of the sleeping periods, e.g. having the first sleeping period longer, leaving less time for wake ups and less active periods during the off period of eDRX. This suggested solution does not specify which sleeping period performs better when extended. The suggested solution in [3] extends the time of the first sleeping period, but it does show any result if the time of any other sleeping period was extended. This is for further study 

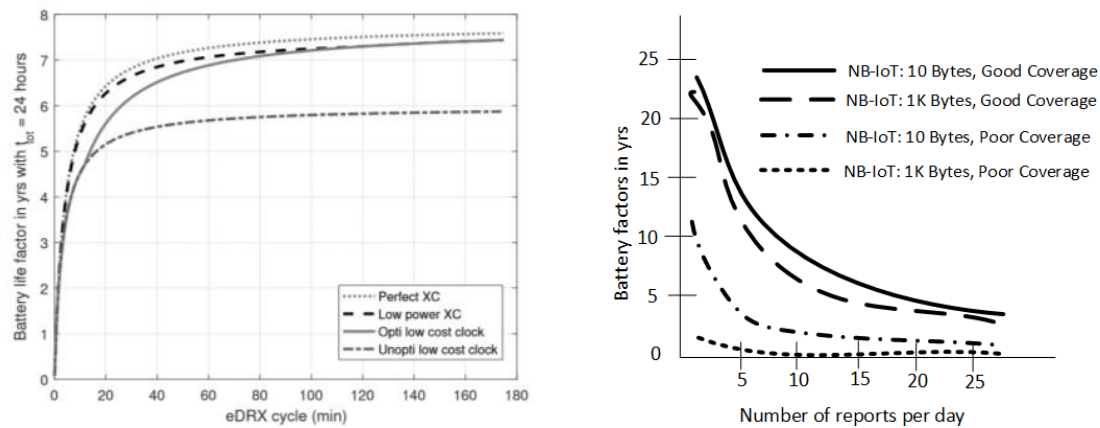

Figure 7, battery life time under different eDRX values Fig. 8: Battery life time for under different scenarios

Figure 7, shows the battery life for NB-IoT 164dB MCL (Maximum Coupling Loss) [3], as a function of eDRX for a data report of 200 bytes with 24 hours interval using different clocks, where it is seen that the lowest battery life is achieved with the unoptimized low power clock Figure 8, shows the battery life as a function of a number of reports per day [3], where it is seen that the best performance is when the number of transmitted reports per day is very low and the size of the data to be transmitted is small. If the size of the report is 10 bytes and it is transmitted once a day under poor coverage, the life battery can reach up to 8 years. In good coverage this can go even over 20 years

\section{Conclusion}

In this paper I highlighted the key features and advantages of Network Slicing as a virtualisation concept for different services and applications and NB-IoT as an underlying radio technology for IoT network slices. The emphasise in this paper was put on the importance of NB-IoT and its applicability for specific use cases and vertical sectors, such as utilities sector and smart cities, and on the evaluation of NB-IoT performance in terms of power consumption and battery life improvement as key IoT requirements.

In terms of the power saving for IoT devices, I analysed the ways to improve the eDRX mechanism and I described the impact this improvement will have for different eDRX values. Based on these analyses, I showed that the battery life time for NB-IoT varies based on the clocks used for frequency synchronisation and how the battery life can reach up to 8 years in a poor coverage.

My analysis also showed that NB-IoT's best performance are when the length of data and the number of messages sent are small and in this case NB-IoT performs better than any other technology. Overall, the performance of NB-IoT depends on a number of factors, such as data length, bandwidth, RF module, report frequency, maximal coupling loss and latency. NB-IoT is not the best low-cost wide area network if very low latency and large data transmission is required. However, given that IoT sensors most of the time are simple and support low-rate applications in medium to poor coverage scenarios, this makes NB-IoT a good fit.

As for the future work, additional improvements to eDRX are needed and will continue working on this and also will analyse the performance of PSM in conjunction with eDRX to further reduce the IoT power consumption. 


\section{References}

1. Feltrin L., Condoluci M., Mahmoodi T., Dohler M., Verdone R.: NB-IoT:

Performance Estimation and Optimal Configuration,

https://nms.kcl.ac.uk/toktam.mahmoodi/files/EW-NBiot-2018.pdf, 2018

2. Chen M., Miao Y., Hao Y. and Hwang K.: Narrow Band Internet of Things, IEEE, September 2017; https://ieeexplore.iee.org/document/8038776

3. El Soussi M., Zand P., Pasveer F., and Dolmans G.,: Evaluating the Performance of eMTC and NB-IoT for Smart City Applications, November 2017 https://arxiv.org/pdf/1711.07268.pdf

4. GSMA: An Introduction to Network Slicing, 2017; https://www.gsma.com/futurenetworks/wp- content/uploads/2017/11/GSMA-AnIntroduction-to-Network-Slicing.pdf

5. GSMA: Network Slicing: Use Case Requirements; 2018;

6. https://www.gsma.com/futurenetworks/wp- content/uploads/2018/06/NetworkSlicing-Use-Case-Requirements-Final-.pdf

7. Geng L., Dong J., Bryant S., Makhijani K.,Galis A., de Foy X., Kuklinks S.: Network Slicing Architecture, https://tools.ietf.org/id/draft-geng-netslices-architecture-01.html, December 2017

8. 3GPP TS 23.682: "Architecture enhancements to facilitate communications with packet data networks and applications; (Release 13)"

9. 3GPP TS 23.401: "General Packet Radio Service (GPRS) enhancements for Evolved Universal Terrestrial Radio Access Network (E-UTRAN) access; (Release 13)"

10. 3GPP TS 22.368: "Service requirements for Machine-Type Communications (MTC); (Release 13)"

11. 3GPP TR 23.720: "Study on architecture enhancements for Cellular Internet of Things (Release 13)" 